\title{
FLECH: Fuzzy Logic Based Energy Efficient Clustering Hierarchy for Nonuniform Wireless Sensor Networks
}

\author{
Baranidharan Balakrishnan and Santhi Balachandran \\ School of Computing, SASTRA University, Thanjavur, Tamil Nadu, India \\ Correspondence should be addressed to Baranidharan Balakrishnan; baranier85@gmail.com
}

Received 15 July 2016; Revised 25 October 2016; Accepted 15 December 2016; Published 20 February 2017

Academic Editor: Xianfu Lei

Copyright (C) 2017 Baranidharan Balakrishnan and Santhi Balachandran. This is an open access article distributed under the Creative Commons Attribution License, which permits unrestricted use, distribution, and reproduction in any medium, provided the original work is properly cited.

\begin{abstract}
Lifetime of Wireless Sensor Network (WSN) is an important issue which affects its implementation in various real time applications. The major factor behind the energy consumption in WSN is its data collection mechanism. The direct data transmission from each sensor node to the Base Station (BS) consumes more energy than other alternatives. Also it is unnecessary, due to redundant data transmission because of geographically closer nodes. Clustering is the best data collection architectural model for WSN since it takes care of in-network processing which handles redundant data within the network. The techniques used for the network having uniform node distribution are not suitable for the networks which have nonuniformly distributed nodes. This paper contributes a novel clustering algorithm: Fuzzy Logic Based Energy Efficient Clustering Hierarchy (FLECH) for nonuniform WSN. The clusters in FLECH are created using proper parameters which increases the lifetime of the WSN. Fuzzy logic in FLECH is wisely used to combine important parameters like residual energy, node centrality, and distance to BS for electing best suitable nodes as $\mathrm{CH}$ and increases the network lifetime. FLECH performance is verified in different scenarios and the results are compared with LEACH, CHEF, ECPF, EAUCF, and MOFCA. The simulation results clearly indicate the lifetime increase by FLECH over other algorithms and its energy conservation per round of data collection in the network.
\end{abstract}

\section{Introduction}

Wireless Sensor Networks (WSN) are made up of hundreds to thousands number of small miniature sensor nodes interconnected with each other. A sensor node is made up of three subsystems such as sensing unit, processing unit, and communication unit [1]. The sensing unit has different types of sensors to measure various atmospheric parameters such as temperature, pressure, light, and sound. Typically, a sensor node is equipped with more than one type of sensor. The processing unit consists of a microcontroller which processes the data generated by the sensing unit. The communication unit is made up of a transceiver chip along with an antenna for enabling data communication among the neighbour sensor nodes or directly with the BS. Apart from the above-mentioned subsystems, a sensor node has a battery unit which is mostly a pair of alkaline batteries for providing power supply to all the above-mentioned subsystems. In terms of energy consumption, communication unit consumes more energy than other units due to transmission, reception, and amplification of data signals [2]. When the battery of a node gets drained, then the node is called as dead.

Direct data transmissions from individual sensor nodes in the network lead to higher energy consumption among the nodes, redundant data transmissions to the BS, and reduced network lifetime. To the best of our knowledge, clustering is the best suitable architecture model for solving the above-mentioned problems [3]. The nearby nodes in the Area of Interest (AOI) form a cluster and elect a cluster head $(\mathrm{CH})$ for managing the clustering activities. A $\mathrm{CH}$ is having the responsibilities like collecting information from the members, aggregating the received information, and communicating aggregated information to central Base Station (BS). Lots of clustering algorithms are being proposed for WSN which elects $\mathrm{CHs}$ through pure probabilistic approach or a weight based approach. Some protocols elect CHs by blending the above two approaches. Both, pure probabilistic 


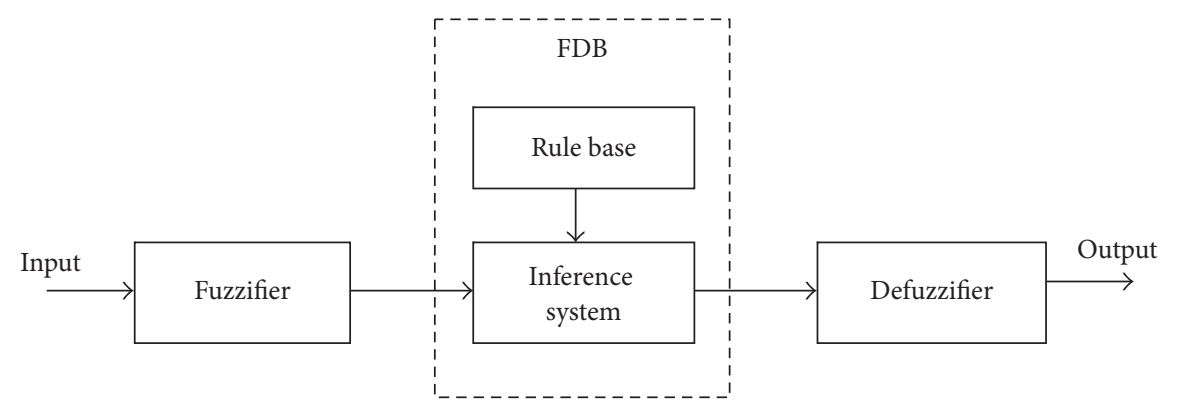

FIGURE 1: Basic block diagram of a fuzzy system.

and weight based approaches fail in achieving an increased lifetime of the network. The probabilistic approach elects $\mathrm{CHs}$ without considering the various lifetime parameters such as energy level, number of neighbour nodes, and intra- and intercluster distance. Due to these reasons, nonsuitable nodes get elected as CHs which affects the lifetime of the WSN. Weight based approach elects $\mathrm{CH}$ s by considering the abovementioned lifetime parameters, but it leads to election of the same nodes as CHs frequently and leads to First Node Die (FND) soon. Since stability of the network is calculated using FND metrics, it should be increased as far as possible. It is found that the algorithms which blend probabilistic approach and weight based approach give better results than following any one approach [4].

Fuzzy Approach. Various computational intelligence (CI) techniques [5] like neural network (NN), Particle Swarm Optimization (PSO), Genetic Algorithms, and so forth are widely used in WSN for various issues. Generally fuzzy logic (FL) $[6,7]$ is used to solve uncertainties in the system. By using FL, a system can be optimized without the need of complete information about it.

There are three significant parts in FL as shown in Figure 1. It consists of fuzzifier, fuzzy decision block (FDB), and defuzzifier block. FDB is made up of rule base and fuzzy inference system. Fuzzifier block converts the given crisp input value into the appropriate fuzzy linguistic variable. Based on the rule base, FDB block will map the set of input linguistic variables to the output linguistic variables. Finally, defuzzifier block converts the fuzzy output variable into the crisp output using a suitable defuzzification method.

Fuzzy Logic Based Energy Efficient Clustering Hierarchy (FLECH), a new clustering algorithm for nonuniform WSN using the fuzzy approach, is proposed in this paper. FLECH uses residual energy, node centrality, and distance to BS to compute the chance of electing a node as $\mathrm{CH}$. Compared with other fuzzy based clustering algorithms, it blends probabilistic approach and weight based approach in the right manner to elect $\mathrm{CH}$ in the network.

The clustering algorithms for WSN are briefly surveyed in Section 2. In Section 3, the proposed algorithm FLECH is described in detail; Section 4 explains the experimental setup and Section 5 gives an analysis over the results.

\section{Related Works}

Low Energy Adaptive Clustering Hierarchy (LEACH) [8] is a distributed clustering algorithm for WSN. It has two phases: setup phase and steady state phase. At first, in the setup phase, each node will decide itself to act as $\mathrm{CH}$ or not in the current round. Factors such as the number of times a node got elected as $\mathrm{CH}$ in the last $r$ rounds and the percentage of desired $\mathrm{CHs}$ will influence the decision. Each node selects a value between 0 and 1 . If the value is less than $T(m)$ as mentioned in (1), the node elects itself as $\mathrm{CH}$ and advertises among its neighbours. In (1), $m$ denotes the sensor node, desired $\mathrm{CH}$ percentage on the network is denoted by $p$, and set of non-CH in the last $r$ rounds is represented by set $G$ :

$$
T(m)= \begin{cases}\frac{p}{1-p \times(r \bmod (1 / p))}, & m \in G \\ 0 & \text { otherwise. }\end{cases}
$$

Remaining nodes join the nearest $\mathrm{CH}$ nodes by sending Join_Req message. Each $\mathrm{CH}$ node generates separate Time Division Multiple Access (TDMA) schedule for their newly formed clusters. In steady state phase, data generated by member nodes are forwarded to their $\mathrm{CH}$ in the allotted time slots. Each $\mathrm{CH}$ aggregates the received data and forwards the aggregated data to the BS. In LEACH, the probabilistic way of electing CHs results in the election of unsuitable nodes as $\mathrm{CHs}$ which affects the lifetime of the network.

In Hybrid Energy Efficient Distributed (HEED) protocol [9], CHs are elected based on the residual energy of the nodes. Whenever there is a tie between two nodes because of the equal residual energy level, the number of neighbour nodes, that is, node degree or distance with the neighbour nodes, is used for $\mathrm{CH}$ election. Due to the consideration of residual energy and node degree for $\mathrm{CH}$ election, HEED [9] provides better performance than LEACH. Equation (2) depicts the $\mathrm{CH}$ probability of a node in HEED. $E_{\text {Residual }}$ represents residual energy, $E_{\max }$ represents the initial maximum energy of a node, and $C_{\text {Prob }}$ is the random number between 0 and 1 in the following:

$$
\mathrm{CH}_{\text {Prob }}=C_{\text {Prob }} \times \frac{E_{\text {Residual }}}{E_{\text {max }}} .
$$

Gupta et al. [10] proposed the implementation of fuzzy logic for electing $\mathrm{CHs}$ in WSN. Three input parameters, 
residual energy, node degree, and node centrality, are used for $\mathrm{CH}$ election. Like in other centralized algorithms, all nodes have to communicate their current residual energy frequently to BS which again increases communication cost. Also the proposed work suffers from scalability issue which is not suitable for large scale WSN.

Cluster head election using fuzzy logic (CHEF) [11] is a distributed clustering algorithm using fuzzy logic approach for WSN. During the start of a round, CHEF elects tentative $\mathrm{CHs}$ using probability approach. The final $\mathrm{CHs}$ are elected from tentative $\mathrm{CH}$ list using residual energy and local distance of the nodes. The sum of distance from all one-hop neighbours to a node $x$ is the local distance of the node $x$. Fuzzy if-then rules are used to evaluate the fuzzified input values. The output variable chance is the potentiality of a node to act as $\mathrm{CH}$. Sensor nodes which got higher chance value will be finally elected as $\mathrm{CH}$. The input fuzzy variable local distance is not a suitable variable for all network sizes and because of this, CHEF suffers a lot in network size apart from $200 \mathrm{~m} \times 200 \mathrm{~m}$.

Energy aware distributed dynamic clustering protocol using fuzzy logic (ECPF) [12] is another fuzzy approach based algorithm for WSN. ECPF has two phases: setup and steady state phase. $\mathrm{CH}$ election and cluster formation will happen in setup phase. TDMA frame generation and data collection happen during the steady state phase. Node degree and node centrality are the two input variables and fuzzy cost is the output variable. Each node in the network will be waiting for a delay time which is inversely proportional to its residual energy. After expiration of delay time, if a node does not receive any tentative_ $\mathrm{CH}$ message from its nearby nodes, then it broadcasts a tentative_CH message containing its id, fuzzy cost, and its status as tentative $\mathrm{CH}$. If there is no other node within its cluster range with lesser fuzzy cost value, it declares itself as the $\mathrm{CH}$ and broadcast final_CH message within its cluster range.

Energy aware unequal clustering using fuzzy approach (EAUCF) $[13,14]$ is also a distributed clustering algorithm for WSN. Residual energy and distance to BS are considered for $\mathrm{CH}$ election in EAUCF. Initially during a round, tentative $\mathrm{CHs}$ are chosen by using random number generation. Fuzzy inference system (FIS) in EAUCF is used to obtain competition radius for the tentative $\mathrm{CH}$ nodes. Each tentative $\mathrm{CH}$ will broadcast its residual energy and check the existence of any other tentative $\mathrm{CH}$ node within its competition radius. If two such tentative $\mathrm{CHs}$ are present within the competition radius of either one node, the nodes having lesser residual energy will quit from $\mathrm{CH}$ competition. In $\mathrm{CH}$ election, an important parameter like node degree is not considered which may lead to election of $\mathrm{CH}$ with fewer and distant neighbours. This results in higher intracluster communication cost and reduces the lifetime of the WSN.

A multiobjective fuzzy clustering algorithm (MOFCA) [15] is a distributed clustering algorithm for WSN. FIS in MOFCA uses residual energy, distance to sink, and node density to compute the competition radius of tentative $\mathrm{CH}$. Like in EAUCF, at the start of a round tentative $\mathrm{CHs}$ are elected using randomized approach. Then the competition radius of each tentative $\mathrm{CH}$ is computed using its FIS. Finally, all tentative $\mathrm{CHs}$ check whether any other tentative $\mathrm{CHs}$ exist within its competition radius, if so, it withdraws from the $\mathrm{CH}$ competition or else it elects itself as $\mathrm{CH}$. Particularly, MOFCA is designed to be suitable for nonuniform network. It uses 27 different output linguistic variables to compute the competition radius.

Though the above presented algorithms prove improvement in increasing lifetime, yet its performance in nonuniform node deployment is poor. The nonuniform node distributions have a dense concentration of nodes in some areas and scarce number of nodes in other parts of AOI. Though both the fuzzy algorithms CHEF and ECPF elect the $\mathrm{CH}$ based on a weight value generated through the fuzzy engine, the $\mathrm{CH}$ role is found to be not properly rotated among the nodes in nonuniform network. In CHEF, local distance parameter is not a suitable input parameter for electing CHs. When a node is having too many numbers of neighbours, local distance also increases, which reduces its chance of becoming $\mathrm{CH}$. But it is well proved that nodes which are having more neighbours are more suitable to be elected as $\mathrm{CH}$. In ECPF, the tentative $\mathrm{CHs}$ are elected using residual energy and final $\mathrm{CHs}$ are elected using fuzzy approach. The static parameters such as node degree and node centrality are taken as input for ECPF's FIS for computing fuzzy cost. Here the major problem is that the same set of nodes are having higher chances of frequently getting elected as $\mathrm{CH}$. This affects the stability period of the network with very earlier First Node Die (FND). In the case of EAUCF, residual energy alone decides the election of final $\mathrm{CH}$ from tentative $\mathrm{CH}$. The very important parameter like number of neighbours and the location of $\mathrm{CH}$ within a cluster is not considered in EAUCF. The key features in FLECH are as follows:

(i) It is a fuzzy based distributed algorithm designed for nonuniform node distribution in WSN.

(ii) Fuzzy logic is wisely used to elect the $\mathrm{CHs}$ nodes by taking into account the factors such as residual energy, node centrality, and distance to BS of the nodes.

(iii) The probabilistic approach is blended suitably with weight based approach for electing suitable $\mathrm{CHs}$.

\section{Proposed Algorithm}

FLECH is designed for nonuniform WSN, which ensures proper rotation of $\mathrm{CH}$ among all the eligible nodes in the network.

\subsection{Network Assumptions}

(i) Homogenous network is assumed where all nodes are having equal capabilities in terms of processing power, sensing area, and so forth.

(ii) All nodes are having the same energy level at the time of deployment.

(iii) The nodes are static after deployment in their AOI.

(iv) Received Signal Strength Indicator (RSSI) is used to calculate the distance between nodes. 
(v) After deployment, all nodes broadcast a HELLO message which contains its ID within its communication radius $R_{c}$. Through this HELLO message only, the number of neighbours and their distance are calculated by each node separately.

(vi) The BS also broadcasts a HELLO message in which its location details are attached.

(vii) Energy depletion alone is the reason behind node death.

3.2. Fuzzy System in FLECH. Residual energy, node centrality, and distance to $B S$ are the input variables in FLECH.

(i) Residual Energy. It is an important factor for electing a node as $\mathrm{CH}$ since a $\mathrm{CH}$ node has to spend more energy than a member node. A CH node collects data from members, aggregates the collected data, and communicates it to BS. So, competent energy level is required for a $\mathrm{CH}$ for executing the above-mentioned activities.

(ii) Node Centrality (NC). Total number of one-hop adjacent nodes within $R_{c}$ of a node is called node degree. Node centrality is a factor which determines how a node is located in the middle among its neighbours. Lower $\mathrm{NC}$ value gives more chance of electing a node as $\mathrm{CH}$ :

$$
\mathrm{NC}=\frac{\sqrt{\sum_{i=1}^{\mathrm{ND}} \mathrm{dist}_{i}{ }^{2} / \mathrm{ND}}}{\text { Ntk_Dimension }} .
$$

In (3) ND (node degree) corresponds to number of neighbours within the communication radius $R_{c}$ of a node and Ntk_Dimension value is " $M$ " in $M \times M$ field area and dist $_{i}{ }^{2}$ represents distance with the $i$ th neighbor node. That is, in $100 \mathrm{~m} \times 100 \mathrm{~m}$ field area, Ntk_Dimension is 100 and $200 \mathrm{~m}$ $\times 200 \mathrm{~m}$ field area and Ntk_Dimension is 200 .

(iii) Distance to BS. The energy consumption for transmitting data increases with the increase in distance between transmitter and receiver nodes. From an energy conservation perspective, the distance between $\mathrm{CH}$ and $\mathrm{BS}$ should be minimized:

$$
\begin{aligned}
\text { Distance to } \mathrm{BS} & =\frac{d_{i}}{\alpha \cdot \text { Ntk_Dimension }}, \\
\alpha & =\frac{d_{\max }}{\text { Ntk_Dimension }} .
\end{aligned}
$$

In (4) $d_{i}$ is the distance between node $i$ and the BS, $d_{\max }$ is the maximum distance between a node in the network and the BS, and $\alpha$ is the network dimension specific constant.

FLECH has one output variable Chance.

(i) Chance. It is a crisp output value based on which $\mathrm{CH}$ capability of a node is determined. The higher chance value represents higher chance of a node in getting elected as a $\mathrm{CH}$.

Low, Medium, and High are the fuzzy linguistic variables for residual energy as shown in Figure 2. Close, Reachable, and Distant for node centrality and Nearby, Average, and Far for

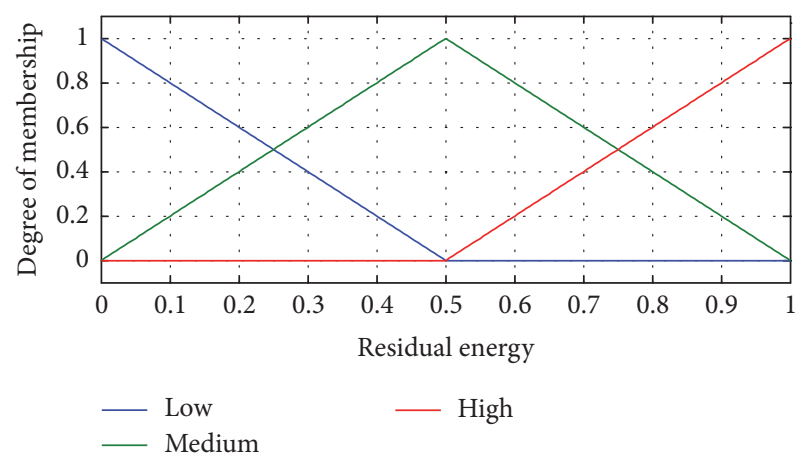

Figure 2: Fuzzy set for the input variable residual energy.

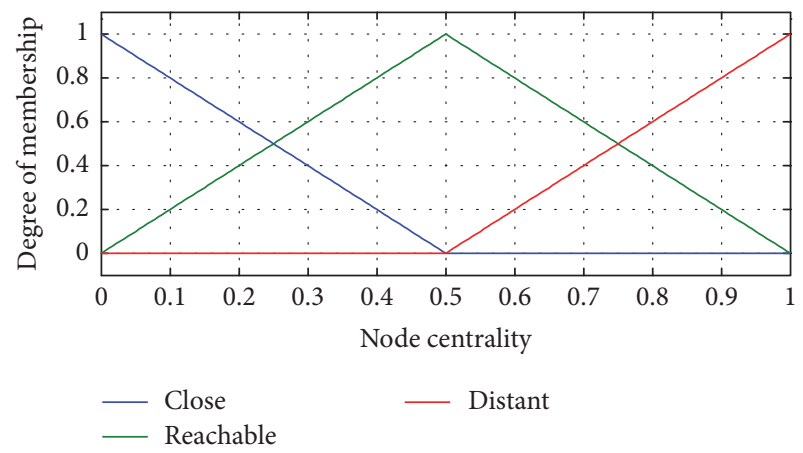

Figure 3: Fuzzy set for the input variable node centrality.

distance to BS are shown in Figures 3 and 4, respectively. Low, High, Close, Distant, Nearby, and Far follow trapezoidal membership function, whereas Medium, Reachable, and Average follow triangular membership function.

The output variable chance has nine output linguistic variables and they are very low, low, rather low, low medium, medium, high medium, rather high, high, and very high. In this very low and very high follow trapezoidal membership function. The remaining output linguistic variables follow triangular membership function. Figure 5 shows the chance membership functions.

The crisp input values are converted into fuzzy linguistic variable based on the given membership function in FIS. Then the fuzzy if-then rules developed based on Mamdani [16] method are used to map the input variables to appropriate fuzzy output variables. In total, 27 fuzzy if-then rules are presented in Table 1 . The crisp output values are obtained using Center of Area (CoA) method.

3.3. Clustering Process. FLECH has two operative phases: (i) cluster formation phase and (ii) data collection phase.

3.3.1. Cluster Formation Phase. At the start of a round, each node has to generate a random value between 0 and 1 . The nodes whose generated random value is less than the predetermined threshold value $P_{\text {thres }}$ are elected as a probationary $\mathrm{CH}$ node. The probationary $\mathrm{CH}$ nodes will compute their chance value using FIS and broadcast Probationary_CH message to all nodes coming under their communication 
TABLE 1: FLECH fuzzy if-then rules.

\begin{tabular}{|c|c|c|c|c|}
\hline S. number & Residual energy & Node centrality & Distance to BS & Chance \\
\hline 1 & Low & Close & Nearby & Rather low \\
\hline 2 & Low & Reachable & Nearby & Low \\
\hline 3 & Low & Distant & Nearby & Very low \\
\hline 4 & Low & Close & Average & Rather low \\
\hline 5 & Low & Reachable & Average & Low \\
\hline 6 & Low & Distant & Average & Very low \\
\hline 7 & Low & Close & Far & Rather low \\
\hline 8 & Low & Reachable & Far & Low \\
\hline 9 & Low & Distant & Far & Very low \\
\hline 10 & Medium & Close & Nearby & Rather medium \\
\hline 11 & Medium & Reachable & Nearby & Medium \\
\hline 12 & Medium & Distant & Nearby & Low medium \\
\hline 13 & Medium & Close & Average & Rather medium \\
\hline 14 & Medium & Reachable & Average & Medium \\
\hline 15 & Medium & Distant & Average & Low medium \\
\hline 16 & Medium & Close & Far & Rather medium \\
\hline 17 & Medium & Reachable & Far & Medium \\
\hline 18 & Medium & Distant & Far & Low medium \\
\hline 19 & High & Close & Near & Very high \\
\hline 20 & High & Reachable & Near & High \\
\hline 21 & High & Distant & Near & Rather high \\
\hline 22 & High & Close & Average & Very high \\
\hline 23 & High & Reachable & Average & High \\
\hline 24 & High & Distant & Average & Rather high \\
\hline 25 & High & Close & Far & Very high \\
\hline 26 & High & Reachable & Far & High \\
\hline 27 & High & Distant & Far & Rather high \\
\hline
\end{tabular}

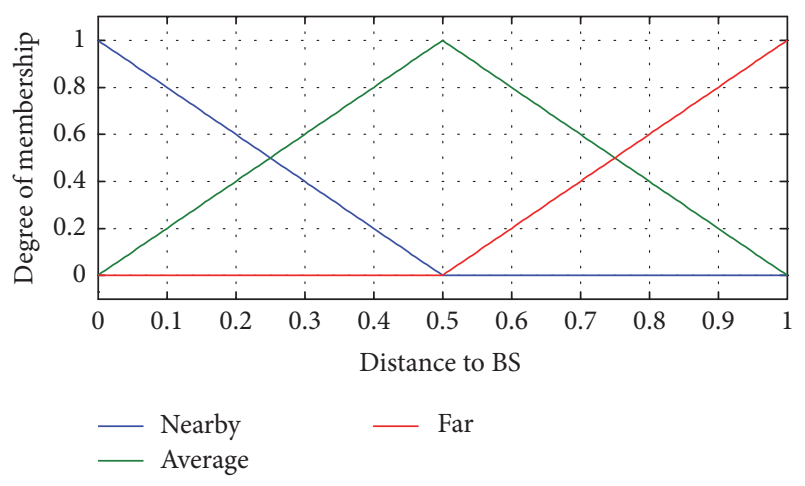

FIGURE 4: Fuzzy set for the input variable distance to BS.

radius $R_{c}$. The Probationary_CH message will contain details such as node id and chance value. If a probationary $\mathrm{CH}$ receives Probationary_ $\mathrm{CH}$ message with higher chance than its own, it withdraws from $\mathrm{CH}$ competition by broadcasting Quit_CH message; otherwise it broadcasts the Elected_CH message to all nodes within radius $R_{c}$. Non- $\mathrm{CH}$ nodes join the nearest $\mathrm{CH}$ by sending Join_Req message. Finally, the newly elected $\mathrm{CH}$ nodes generate the TDMA schedule for their members and broadcast it.

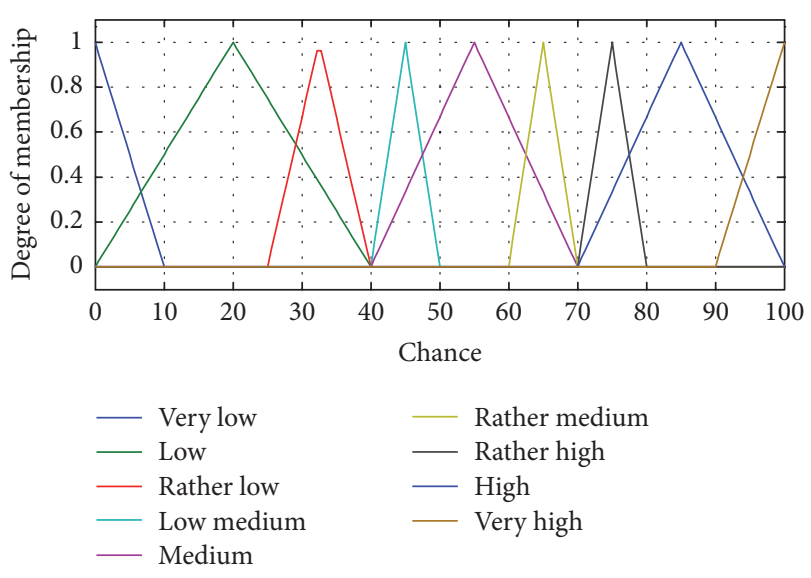

FIGURE 5: Fuzzy set for the output variable chance.

3.3.2. Data Collection Phase. The newly elected CHs will be broadcasting TDMA schedule to its members. Then, member nodes send the data to their respective $\mathrm{CH}$ only during the allotted time slots. In other time slots, they are in the sleep state to conserve energy. The $\mathrm{CH}$ nodes after collecting data from all its members aggregate into a single data message. 


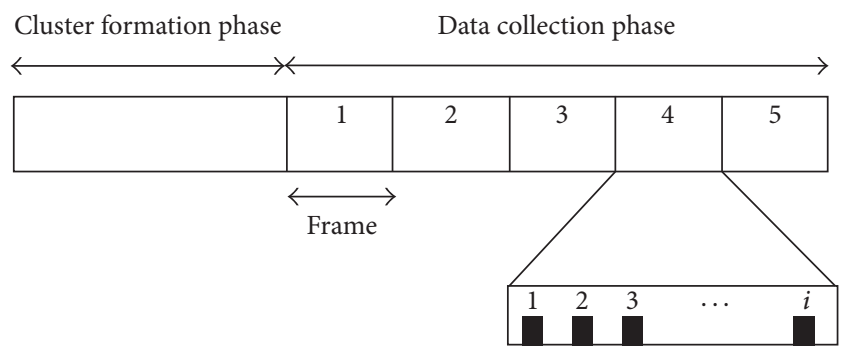

Figure 6: Phases in FLECH.

The aggregated data will be finally reported to BS from $\mathrm{CHs}$. Figure 6 depicts the phases in FLECH. During a frame, a member node will transmit data to $\mathrm{CH}$ only once. All the $\mathrm{CHs}$ should be in wake-up state all through the time unlike member nodes. All these activities of $\mathrm{CH}$ lead to increased energy consumption. In order to achieve balanced energy consumption, $\mathrm{CH}$ role is rotated among the nodes. Equation (5) shows the total energy spent during data collection from sensor nodes.

$$
\begin{aligned}
\mathrm{TE} & =E_{\text {intra-cluster }}+E_{\text {inter-cluster }}, \\
E_{\text {intra-cluster }} & =E_{\text {mem-CH }}+E_{r x}+E_{\mathrm{DA}} .
\end{aligned}
$$

In (6), $E_{\text {mem-CH }}$ is the energy for data transmission from members of the corresponding $\mathrm{CH}, E_{r x}$ represents data reception energy, and $E_{\mathrm{DA}}$ represents data aggregation energy in $\mathrm{CHs}$ nodes.

$$
E_{\text {mem-CH }}=\sum_{i=1}^{k} \sum_{j=1}^{m} E_{T x}\left(j, \mathrm{CH}_{i}\right) .
$$

In (7), $k$ represents the number of clusters, $m$ is the number of members within a cluster, and $E_{T x}\left(j, \mathrm{CH}_{i}\right)$ is the transmission energy cost from node $j$ to its $\mathrm{CH}$ in the $i$ th cluster. Equation (8) represents the total data aggregation energy in the network

$$
E_{\mathrm{DA}}=n \cdot m \cdot E_{\text {perdatabit }},
$$

where $n$ is the number of bits and $E_{\text {perdatabit }}$ represents the aggregation energy for a single data bit in (8). In (9) the intercluster energy consumption is represented.

$$
E_{\text {inter-cluster }}=\sum_{i=1}^{k}\left(E_{T x}\left(\mathrm{CH}_{i}, \mathrm{BS}\right)\right) \text {. }
$$

Equation (10) represents the energy consumption at a $\mathrm{CH}$ node under the condition when $\mathrm{CH}$ is merely acting as a gateway node for the members and does not generate any data on its own. $N$ denotes total number of nodes in the network, $k$ is the number of clusters, $E_{\text {elec }}$ is the electronics energy, and $d$ is the distance between transmitter and receiver in the following:

$$
\begin{aligned}
E_{\mathrm{CH}}= & \left(n \cdot E_{\mathrm{elec}}+n \cdot E_{\mathrm{DA}}\right)\left(\frac{N}{k}-1\right)+n \cdot E_{\mathrm{elec}}+n \\
& \cdot \varepsilon_{m p} \cdot d^{4} .
\end{aligned}
$$

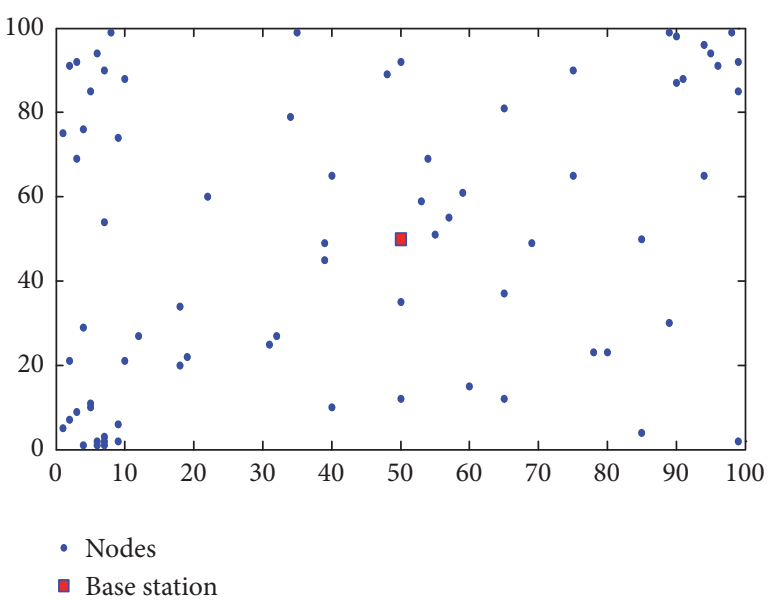

FIGURE 7: Representative network snap shot with BS in the middle of AOI.

Equation (11) represents the energy consumption at a $\mathrm{CH}$ node when the $\mathrm{CH}$ node is also involved in sensing activities and generates the data. In general, BS is assumed to be located outside the AOI and distance between $\mathrm{CH}$ and $\mathrm{BS}$ is larger. So the multipath loss $\varepsilon_{m p}$ is considered for the $\mathrm{CH}$ to $\mathrm{BS}$ communications:

$$
\begin{aligned}
E_{\mathrm{CH}}= & n \cdot E_{\text {elec }}\left(\frac{N}{k}-1\right)+n \cdot E_{\mathrm{DA}}\left(\frac{N}{k}\right)+n \cdot E_{\text {elec }} \\
& +n \cdot \varepsilon_{m p} \cdot d^{4} .
\end{aligned}
$$

The energy consumption at a member node is depicted in (12). Since the distance between a member and its $\mathrm{CH}$ node is minimum, free space loss $\varepsilon_{f s}$ is considered for member to $\mathrm{CH}$ communications. FLECH algorithm is shown in Algorithm 1.

$$
E_{\text {member }}=n \cdot E_{\text {elec }}+n \cdot \varepsilon_{f s} \cdot d^{2} .
$$

\section{Experimental Setup}

The proposed work FLECH is compared with LEACH, CHEF, ECPF, EAUCF, and MOFCA. LEACH is considered since it is the base for all the distributed clustering protocols for WSN. ECPF, CHEF, EAUCF, and MOFCA are used in comparison since these algorithms use fuzzy logic for clustering in WSN. MOFCA is a recent clustering algorithm for nonuniform WSN. Most of the clustering algorithms designed for WSN are found to be suitable for specific network field size. In order to verify the efficiency of FLECH over other clustering algorithms, four different network scenarios are considered. In all of the four network scenarios, two cases are considered: (i) BS is located within AOI, that is, at the centre of AOI, and (ii) BS is located away from AOI. Figures 7 and 8 depict the above two cases.

In all the scenarios, performance of the above-mentioned clustering algorithms is verified by using network size of 100 , 200, 300, and 400 nodes The network scenarios are given below. 


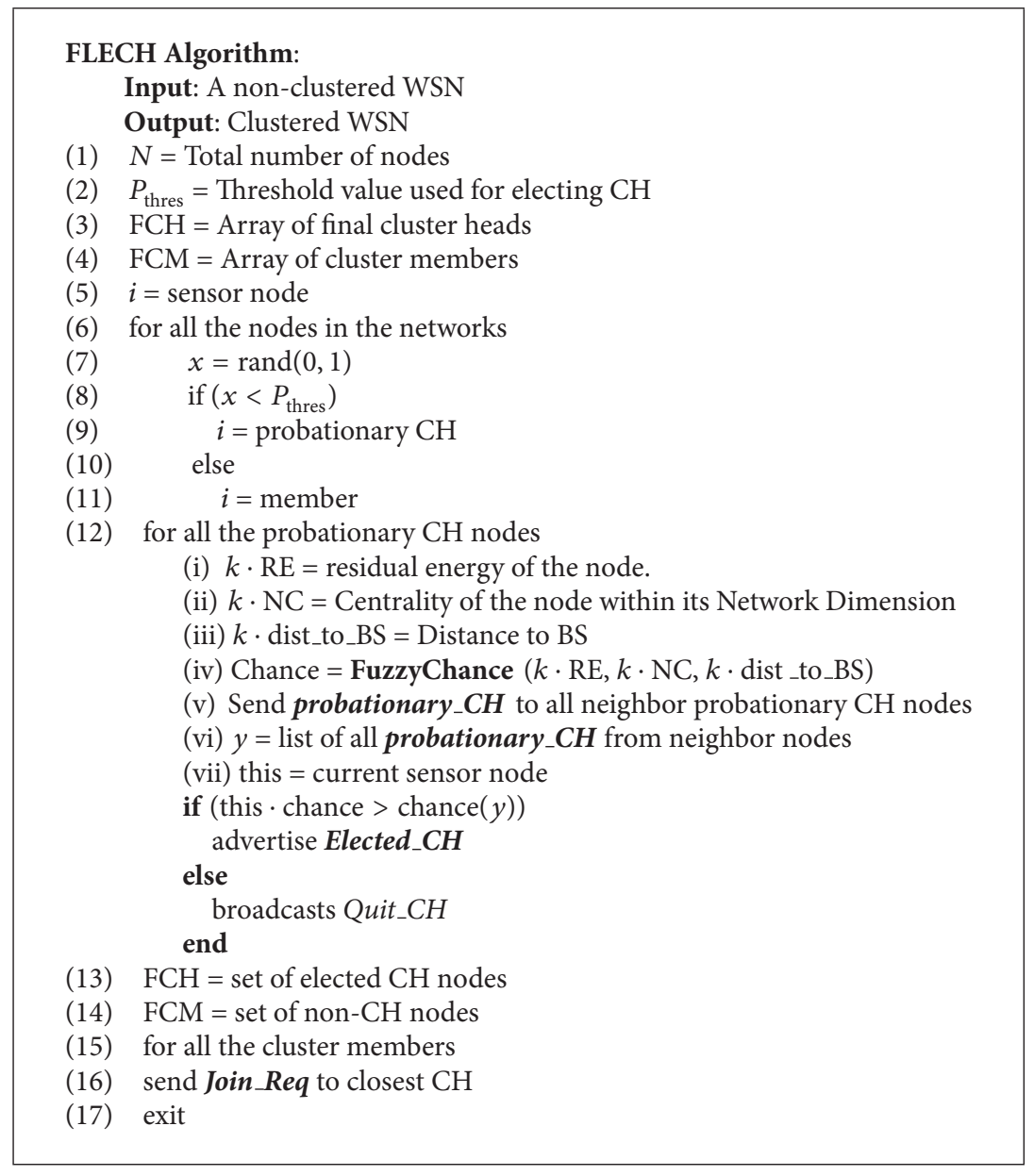

Algorithm 1: FLECH.

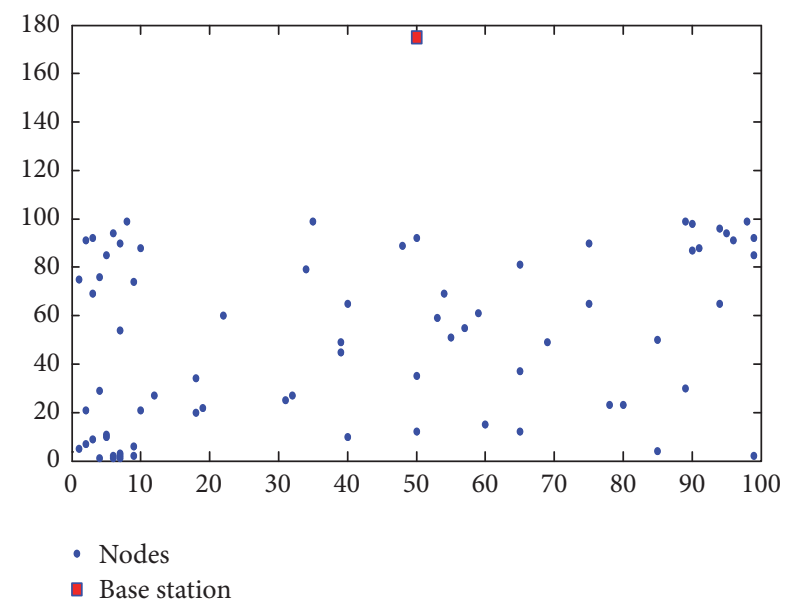

FIGURE 8: Representative network snap shot with BS outside AOI.

Scenario 1. Nodes are randomly deployed over the area of $100 \mathrm{~m} \times 100 \mathrm{~m}$. The BS is located at the coordinate $(50,50)$.

Scenario 2. Nodes are randomly deployed over the area of $100 \mathrm{~m} \times 100 \mathrm{~m}$. The BS is located at the coordinate $(50,175)$.
Scenario 3. Nodes are randomly deployed over the area of $200 \mathrm{~m} \times 200 \mathrm{~m}$. The BS is located at the coordinate $(100,100)$.

Scenario 4. Nodes are randomly deployed over the area of $200 \mathrm{~m} \times 200 \mathrm{~m}$. The BS is located at the coordinate $(100,275)$.

The communication model used in [13] is followed in this experimental setup also:

Transmission energy, $E_{T x}$

$$
= \begin{cases}n \cdot E_{\mathrm{elec}}+\varepsilon_{f s} \cdot d^{2} & d \leq d_{0} \\ n \cdot E_{\mathrm{elec}}+\varepsilon_{m p} \cdot d^{4} & d>d_{0},\end{cases}
$$

Receiving energy, $E_{R x}=n \cdot E_{\text {elec }}$.

In (13), $n$ denotes the number of transmission bits, $E_{\text {elec }}$ is the electronics energy or circuit loss, $\varepsilon_{f s}$ represents power loss in free space propagation model and $\varepsilon_{m p}$ represents the power loss in multipath propagation model, $d$ represents the distance between transmitter and receiver nodes, and $d_{0}$ is the threshold distance for determining whether free space or multipath propagation is going to be followed. Table 2 depicts the simulation values for various parameters. 
TABLE 2: Simulation values.

\begin{tabular}{lc}
\hline Parameters & Values \\
\hline$E_{\text {elec }}$ & $50 \mathrm{~nJ} \mathrm{bit}^{-1}$ \\
$\varepsilon_{f s}$ & $10 \mathrm{pJ} \mathrm{bit}^{-1}$ \\
$\varepsilon_{m p}$ & $0.0013 \mathrm{pJ} \mathrm{bit}^{-1}$ \\
$d_{0}-$ distance & $87 \mathrm{~m}$ \\
Initial energy & $1 \mathrm{~J}$ \\
Data message & $4000 \mathrm{bits}$ \\
Control message & $200 \mathrm{bits}$ \\
Frames per round & 5 \\
\hline
\end{tabular}

The control messages are associated with cluster formation activities. The data messages are the data sent by member nodes to $\mathrm{CH}$ and from $\mathrm{CH}$ to the $\mathrm{BS}$. Data message is of the size of 4000 bits which is much larger than control messages which are only 200 bits. During a frame in the round, the members will be transmitting its data to the $\mathrm{CH}$ in its time slot. At the end of each frame, the aggregated data from $\mathrm{CH}$ will be communicated to the BS. The data aggregation energy is $E_{\mathrm{DA}}=5 \mathrm{~nJ}$ per bit. And in a frame only one data packet will be transmitted from a member to the $\mathrm{CH}$. The transmission and reception cost will be subtracted in the respective node's energy level.

The desired $\mathrm{CH}$ percentage for LEACH is 0.1 in all four scenarios. $P_{\text {opt }}$ in CHEF, EAUCF, and MOFCA is set at 0.3. The threshold value $P_{\text {thres }}$ in FLECH is set as 0.1 since it gives better results. $R_{c}$ of the sensor nodes is set as $25 \mathrm{~m}$ like in MICA motes for all the scenarios. $R_{c}$ is mainly used for finding number of neighbours within that radius and forming the clusters with that as boundary radius. But when a node gets elected as $\mathrm{CH}$, it is capable of sending data to distant points away from $R_{c}$ by increasing its transmission power. So the direct data transmission from $\mathrm{CHs}$ to the $\mathrm{BS}$ is possible. In all the simulations, for all the clustering algorithms, the $\mathrm{CHs}$ are directly transmitting the aggregated data to the BS. All the results presented are based on 100 simulation runs in our simulator. For each scenario and for each case under that scenario, 10 simulation runs are conducted in different network topology and totally 100 simulations are conducted for all four scenarios.

A simulator is developed in MATLAB for comparing the performance of FLECH with other clustering algorithms such as LEACH, CHEF, ECPF, EAUCF, and MOFCA in nonuniform node distribution condition. The nonuniform node deployment is done manually by arranging more number of nodes in certain areas and very less number of nodes in some other areas. The fuzzy inference system (FIS) is built using the toolbox environment in MATLAB for FLECH, MOFCA, EAUCF, ECPF, and CHEF.

\section{Results and Analysis}

The results are analysed with respect to the following two categories.

Network Lifetime. Various metrics are there to denote the lifetime of the WSN. But here, First Node Die (FND) and

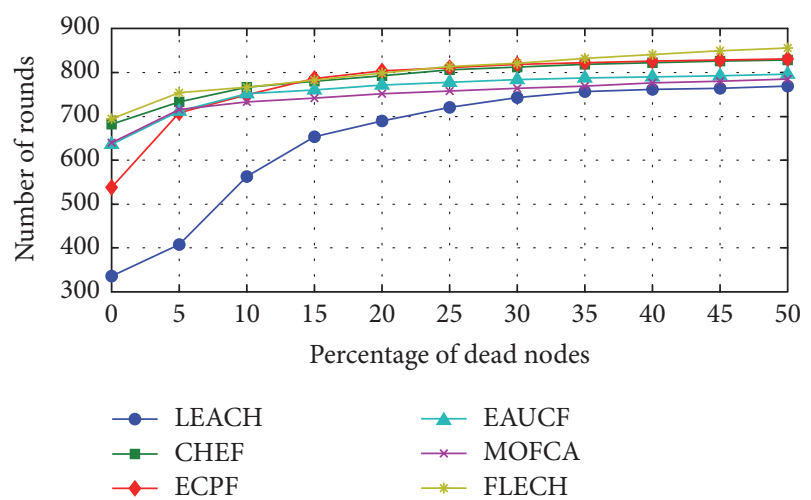

FIGURE 9: Network lifetime in Scenario 1 with 100 nodes.

Half Node Die (HND) are used to compare and analyse the network. FND is the number of completed rounds till the death of the first sensor node in the network and HND is equal to death of $50 \%$ of sensor nodes. FND has been considered since the stability period depends on it. After 50\% of nodes die, the reliability of the network is completely lost. So the lifetime is considered up to HND only.

Average Energy Consumption per Round. The average energy consumption during a round in all the clustering algorithms till First Node Die (FND). The FND is taken since it represents the stability period of the network.

FLECH is evaluated with LEACH, CHEF, ECPF, EAUCF, and MOFCA by FND and HND in all the four scenarios mentioned.

5.1. Network Lifetime. In all the scenarios, ECPF suffers a lot because of pure metric based election of $\mathrm{CH}$ which is not suitable for a nonuniform network. In the case of ECPF, the highly suitable nodes are frequently elected as $\mathrm{CH}$ which causes premature death of certain nodes. $\mathrm{LEACH}$ comes next to ECPF whereas LEACH elects $\mathrm{CH}$ through pure probabilistic way. FLECH gives better improved performance than all other algorithms in all the scenarios and this happened because of consideration of various factors like residual energy, node centrality, and distance to BS for electing a $\mathrm{CH}$ node. FLECH ensures the proper rotation of $\mathrm{CH}$ responsibility among all the nodes through the initial probabilistic way of electing the probationary $\mathrm{CH}$ and metric based final CH election. Also, FLECH is designed in such a way it is suitable for various sizes and scale of the network.

5.1.1. Scenario 1. Figures 9 and 10 depict the network lifetime in Scenario 1 with network size of 100 nodes and 200 nodes, respectively. Comparing with other scenarios, more rounds are achieved in this scenario due to the location of BS at the centre of AOI. FLECH performance is better than all other clustering algorithms because of its appropriate input variables. CHEF achieves increased lifetime than EAUCF, MOFCA, and ECPF. In both EAUCF and MOFCA, the output fuzzy variable is competition radius, where the competition for $\mathrm{CH}$ occurs between tentative $\mathrm{CHs}$ within that competition 


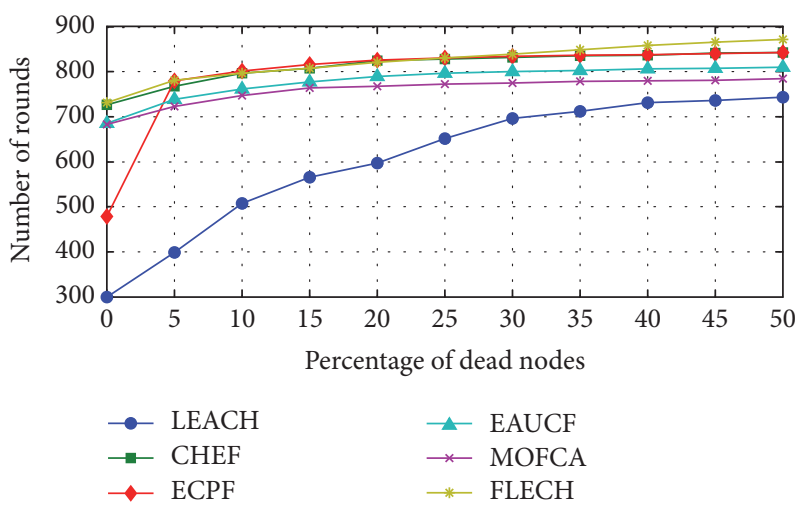

FIGURE 10: Network lifetime in Scenario 1 with 200 nodes.

TABLE 3: FND and HND for 100 and 200 nodes.

\begin{tabular}{lcccc}
\hline \multirow{2}{*}{ Algorithms } & \multicolumn{2}{c}{100 nodes } & \multicolumn{2}{c}{200 nodes } \\
& FND & HND & FND & HND \\
\hline LEACH & 335 & 769 & 300 & 743 \\
CHEF & 682 & 829 & 726 & 842 \\
ECPF & 538 & 831 & 479 & 843 \\
EAUCF & 636 & 796 & 685 & 810 \\
MOFCA & 640 & 785 & 683 & 784 \\
FLECH & 695 & 856 & 731 & 872 \\
\hline
\end{tabular}

radius. But in CHEF and FLECH, the output fuzzy variable is the chance of a tentative $\mathrm{CH}$ to become a $\mathrm{CH}$ node. Also, it is observed from Figures 9 and 10 that increasing the number of nodes in AOI increases FND considerably. But in the case of LEACH and ECPF, an increase in the number of nodes reduces its FND because of their respective pure probabilistic and weight based approaches.

Table 3 depicts the simulation results for Scenario 1. In a 100-node setup, considering FND, FLECH increases network lifetime compared to LEACH by $107 \%$, CHEF by $1.9 \%$, ECPF by $29.1 \%$, EAUCF by $9.27 \%$, and MOFCA by $8.59 \%$. On considering HND, FLECH is better than LEACH by $11.31 \%$, CHEF by $3.25 \%$, ECPF by $3 \%$, EAUCF by $7.53 \%$, and MOFCA by $9.04 \%$.

In a 200-node setup, on considering FND, FLECH is better than LEACH by $143.55 \%$, ECPF by $52.60 \%$, EAUCF by $6.71 \%$, and MOFCA by $8 \%$. On considering HND, FLECH shows improvement compared to LEACH by $17.36 \%$, CHEF by $3.56 \%$, ECPF by $3.44 \%$, EAUCF by $7.65 \%$, and MOFCA by $11.22 \%$.

5.1.2. Scenario 2. This scenario corresponds to location of BS outside the AOI in $100 \mathrm{~m} \times 100 \mathrm{~m}$ setup. As like in Scenario 1, here also two different network sizes of 100 and 200 nodes are considered. Figure 11 shows network lifetime for 100 nodes setup and Figure 12 depicts network lifetime for 200-node setup. FLECH shows increased FND compared to other algorithms in both cases. In case of HND, EAUCF and MOFCA show slight improvement over FLECH. Table 4 depicts the simulation results for Scenario 2.

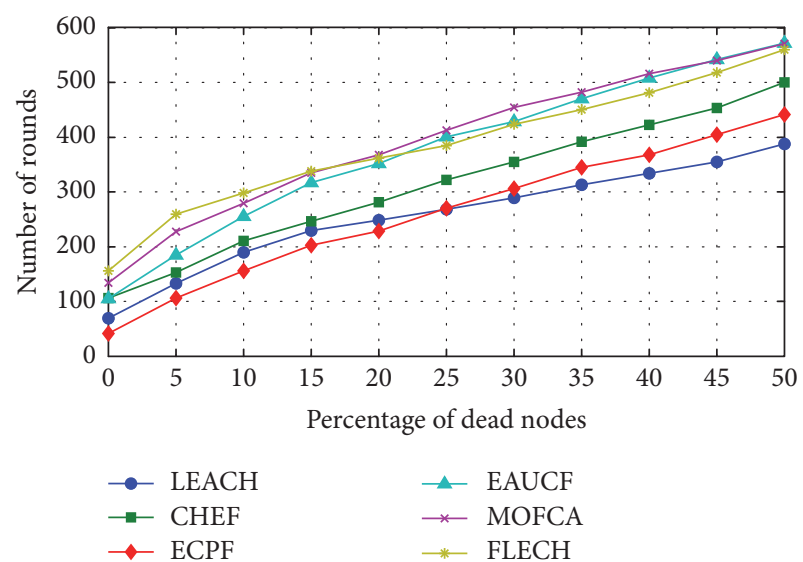

Figure 11: Network lifetime in Scenario 2 with 100 nodes.

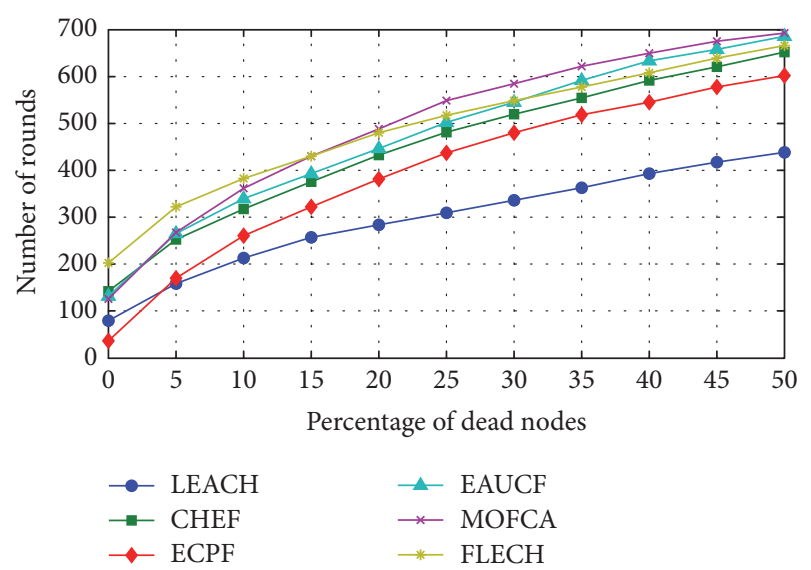

FIGURE 12: Network lifetime in Scenario 2 with 200 nodes.

TABLE 4: FND and HND for 100 and 200 nodes.

\begin{tabular}{lcccc}
\hline \multirow{2}{*}{ Algorithms } & \multicolumn{2}{c}{100 nodes } & \multicolumn{2}{c}{200 nodes } \\
& FND & HND & FND & HND \\
\hline LEACH & 69 & 388 & 80 & 438 \\
CHEF & 106 & 500 & 142 & 652 \\
ECPF & 41 & 441 & 36 & 602 \\
EAUCF & 105 & 572 & 132 & 686 \\
MOFCA & 134 & 571 & 126 & 693 \\
FLECH & 156 & 560 & 203 & 666 \\
\hline
\end{tabular}

In a 100-node setup, considering FND, FLECH increases network lifetime compared to LEACH by $126.08 \%$, CHEF by $47.16 \%$, ECPF by $280.48 \%$, EAUCF by $48.57 \%$, and MOFCA by $16.41 \%$. On considering HND, FLECH is better than LEACH by $44.32 \%$, CHEF by $12 \%$, and ECPF by $26.98 \%$. In HND condition, EAUCF and MOFCA show slightly higher number of rounds than FLECH.

In a 200-node setup, on considering FND, FLECH is better than LEACH by $153.75 \%$, CHEF by $42.95 \%$, ECPF by $463.88 \%$, EAUCF by $53.78 \%$, and MOFCA by $61.11 \%$. On considering HND, FLECH shows improvement compared to LEACH by $52.05 \%$, CHEF by $2.14 \%$, and ECPF by $10.63 \%$. 


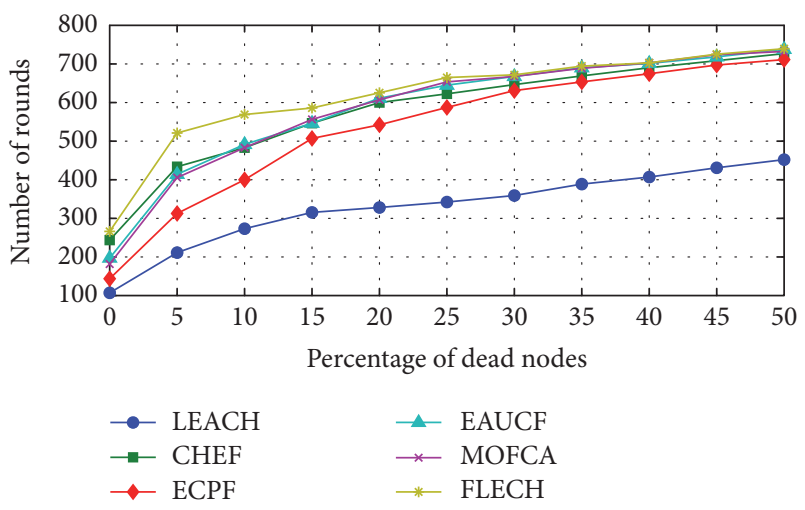

FIGURE 13: Network lifetime in Scenario 3 with 200 nodes.

TABLE 5: FND and HND for 200, 300, and 400 nodes.

\begin{tabular}{lcccccc}
\hline \multirow{2}{*}{ Algorithms } & \multicolumn{2}{c}{200 nodes } & \multicolumn{2}{c}{300 nodes } & \multicolumn{2}{c}{400 nodes } \\
& FND & HND & FND & HND & FND & HND \\
\hline LEACH & 107 & 451 & 155 & 581 & 136 & 614 \\
CHEF & 243 & 726 & 326 & 758 & 321 & 790 \\
ECPF & 143 & 710 & 141 & 758 & 113 & 787 \\
EAUCF & 196 & 738 & 207 & 750 & 280 & 775 \\
MOFCA & 181 & 731 & 229 & 745 & 300 & 762 \\
FLECH & 265 & 739 & 362 & 761 & 417 & 785 \\
\hline
\end{tabular}

Like in 100-node setup, here also EAUCF and MOFCA show higher number of rounds.

5.1.3. Scenario 3. In this scenario, bigger deployment area of $200 \mathrm{~m} \times 200 \mathrm{~m}$ is taken for simulation. In $200 \mathrm{~m} \times 200 \mathrm{~m}$ area, network sizes of 200,300, and 400 nodes are taken for simulations. In Scenario 3, BS is located within the AOI. In order to verify the performance of proposed and existing algorithms in different network sizes, Scenarios 3 and 4 are considered. Figures 13, 14, and 15 depict the network lifetime for 200, 300, and 400 nodes, respectively. In all the network size setups, FLECH shows improved lifetime than other algorithms in FND condition. In HND case, FLECH performance is nearly equal with CHEF, ECPF, EAUCF, and MOFCA. LEACH performance is poor in HND condition compared to all others due to pure probabilistic approach.

Table 5 depicts the simulation results for Scenario 3. In a 200-node setup, considering FND, FLECH increases network lifetime compared to LEACH by $147.66 \%$, CHEF by $9.05 \%$, ECPF by $85.31 \%$, EAUCF by $35.20 \%$, and MOFCA by $46.40 \%$. On considering HND, FLECH is better than LEACH by $63.85 \%$, CHEF by $1.79 \%$, and ECPF by $4.08 \%$. In HND condition, FLECH performance is similar to EAUCF and MOFCA.

In a 300-node setup, on considering FND, FLECH is better than LEACH by $133.54 \%$, CHEF by $11.04 \%$, ECPF by $156.73 \%$, EAUCF by $74.87 \%$, and MOFCA by $58.07 \%$. On considering HND, FLECH shows improvement compared to LEACH by $30.98 \%$. CHEF, ECPF, EAUCF, and MOFCA show similar performance to FLECH.

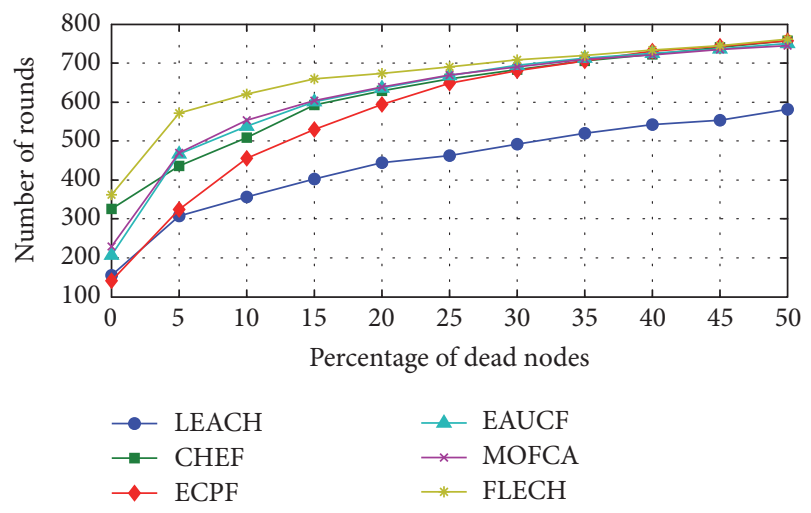

FIGURE 14: Network lifetime in Scenario 3 with 300 nodes.

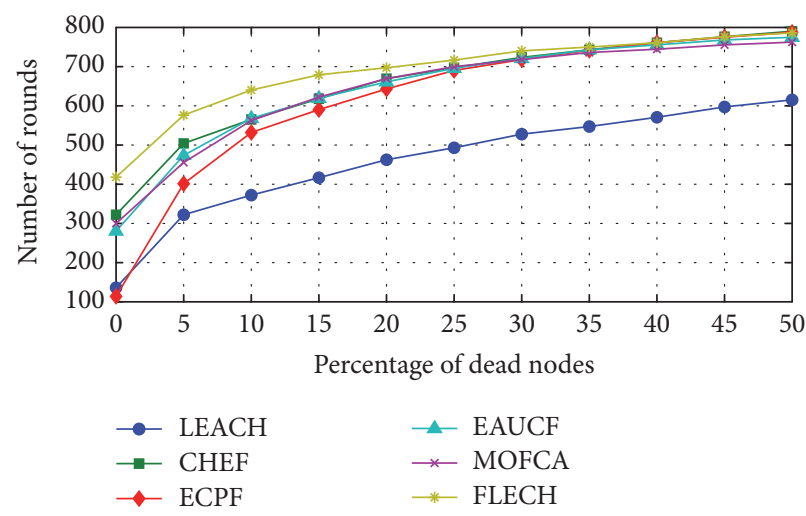

FIgURE 15: Network lifetime in Scenario 3 with 400 nodes.

In a 400-node setup, considering FND, FLECH shows improvement in network lifetime compared to LEACH by $206.61 \%$, CHEF by $29.90 \%$, ECPF by $269.02 \%$, EAUCF by $48.92 \%$, and MOFCA by $39 \%$. On considering HND, FLECH is better than LEACH by $27.85 \%$ and MOFCA by $3.01 \%$. CHEF, ECPF, and EAUCF show similar performance to FLECH.

5.1.4. Scenario 4. Like Scenario 3, in Scenario 4 also deployment area is the same, that is, $200 \mathrm{~m} \times 200 \mathrm{~m}$, but the BS is located outside the AOI in Scenario 4. Comparing with all other scenarios, in this scenario number of rounds will be very less due to long distance BS from the nodes. FLECH performance is better than others in this scenario also. CHEF shows betterment than other fuzzy clustering algorithms such as MOFCA, EAUCF, and ECPF and comes next to FLECH. Pure weight based $\mathrm{CH}$ election affects the network lifetime of ECPF a lot. Figures 16, 17, and 18 depicts the network lifetime of 200-, 300-, and 400-node setup, respectively. Table 6 depicts the simulation results for Scenario 4.

In a 200-node setup, considering FND, FLECH increases network lifetime compared to LEACH by $150 \%$, CHEF by $31.57 \%$, ECPF by $212.5 \%$, EAUCF by $92.30 \%$, and MOFCA by $92.30 \%$. On considering HND, FLECH is better than LEACH by $34.78 \%$, CHEF by $29.16 \%$, ECPF by $88.69 \%$, and EAUCF by 


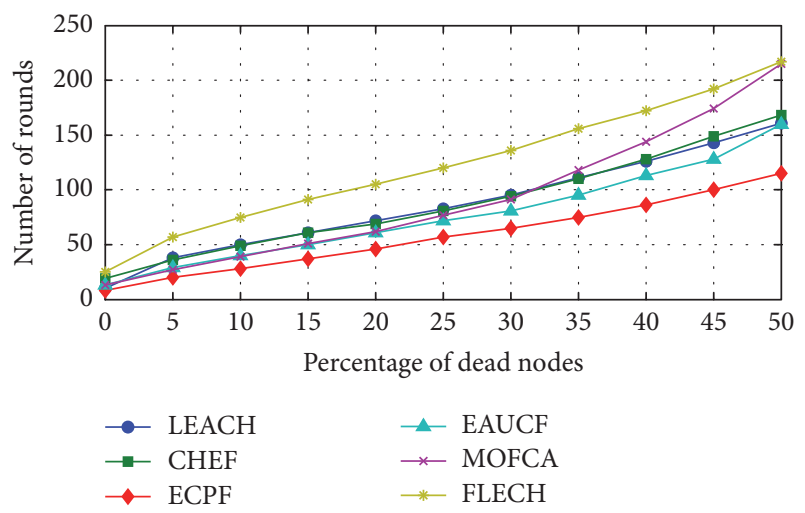

FIgURE 16: Network lifetime in Scenario 4 with 200 nodes.

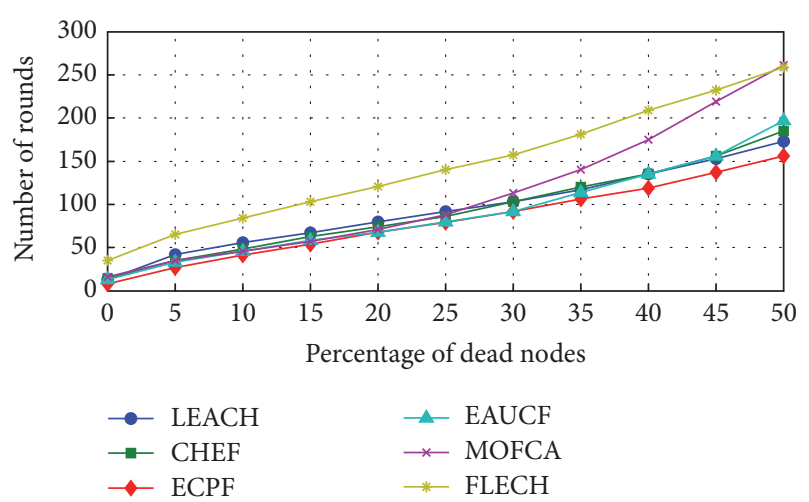

Figure 17: Network lifetime in Scenario 4 with 300 nodes.

TABLE 6: FND and HND for 200, 300, and 400 nodes.

\begin{tabular}{lcccccc}
\hline \multirow{2}{*}{ Algorithms } & \multicolumn{2}{c}{200 nodes } & \multicolumn{2}{c}{300 nodes } & \multicolumn{2}{c}{400 nodes } \\
& FND & HND & FND & HND & FND & HND \\
\hline LEACH & 10 & 161 & 13 & 173 & 17 & 180 \\
CHEF & 19 & 168 & 14 & 185 & 13 & 215 \\
ECPF & 8 & 115 & 8 & 156 & 7 & 197 \\
EAUCF & 13 & 160 & 13 & 197 & 14 & 218 \\
MOFCA & 13 & 215 & 16 & 261 & 13 & 281 \\
FLECH & 25 & 217 & 35 & 259 & 24 & 294 \\
\hline
\end{tabular}

$35.62 \%$. In HND condition, MOFCA performance is similar to FLECH.

In a 300-node setup, on considering FND, FLECH is better than LEACH by $169.23 \%$, CHEF by $150 \%$, ECPF by $337.5 \%$, EAUCF by $169.23 \%$, and MOFCA by $118.75 \%$. On considering HND, FLECH shows improvement compared to $\mathrm{LEACH}$ by $49.71 \%$, CHEF by $40 \%$, ECPF by $66 \%$, and EAUCF by $31.47 \%$. In HND condition, MOFCA shows similar performance to FLECH.

In a 400-node setup, considering FND, FLECH shows improvement in network lifetime than LEACH by $41.17 \%$, CHEF by $84.61 \%$, ECPF by $242.85 \%$, EAUCF by $71.42 \%$, and MOFCA by $84.61 \%$. On considering HND, FLECH is better than LEACH by $63.33 \%$, CHEF by $36.74 \%$, ECPF by $50 \%$, EAUCF by $34.86 \%$, and MOFCA by $4.62 \%$.

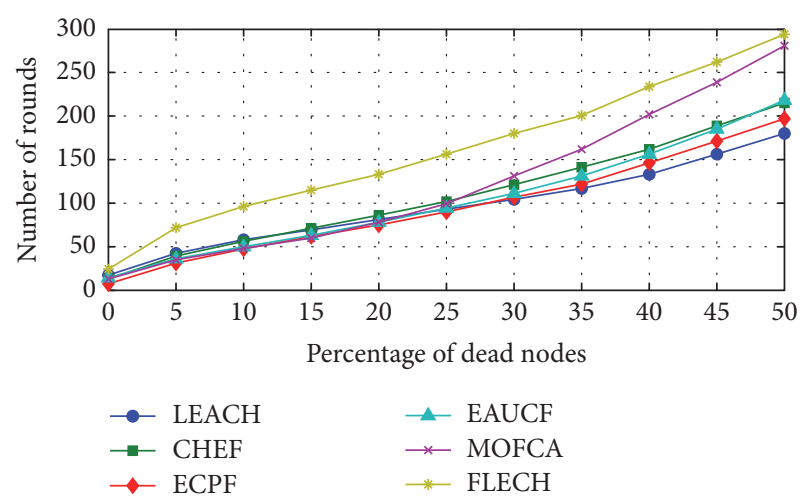

FIGURE 18: Network lifetime in Scenario 4 with 400 nodes.

TABLE 7: Average energy consumption per round in Scenarios 1 and 2.

\begin{tabular}{lcccc}
\hline \multirow{2}{*}{ Algorithms } & \multicolumn{2}{c}{100 nodes } & \multicolumn{2}{c}{200 nodes } \\
& $\begin{array}{c}\text { BS inside } \\
\text { AOI }\end{array}$ & $\begin{array}{c}\text { BS outside } \\
\text { AOI }\end{array}$ & $\begin{array}{c}\text { BS inside } \\
\text { AOI }\end{array}$ & $\begin{array}{c}\text { BS outside } \\
\text { AOI }\end{array}$ \\
\hline LEACH & 0.1137 & 0.26116 & 0.2180 & 0.45584 \\
CHEF & 0.1103 & 0.20882 & 0.2161 & 0.3225 \\
ECPF & 0.1106 & 0.2439 & 0.2169 & 0.35422 \\
EAUCF & 0.1151 & 0.1853 & 0.2285 & 0.30576 \\
MOFCA & 0.1177 & 0.18324 & 0.2331 & 0.3163 \\
FLECH & 0.1098 & 0.1792 & 0.2151 & 0.30292 \\
\hline
\end{tabular}

5.2. Average Energy Consumption per Round. Table 7 depicts the average energy consumption per round by all the clustering algorithms in Scenarios 1 and 2. It includes the cluster formation cost and intracluster and intercluster energy cost for all the frames during a round. Comparing with other clustering algorithms LEACH gives the poor performance because of poor clustering. Most of the times in LEACH, the unsuitable nodes are getting elected as $\mathrm{CH}$ which leads to higher intracluster and intercluster communication cost. It can be observed from the table that the average energy consumption by FLECH is less than other algorithms in all the cases.

Table 8 shows the average energy consumed per round by the clustering algorithms for Scenarios 3 and 4 . Also it is observed from Table 8 that when the number of nodes increases, average energy consumption difference between FLECH and other algorithms also increases which proves that FLECH is scalable with network size than others. All the specified values in Tables 7 and 8 are in Joules.

\subsection{Scalability and Complexity Analysis of FLECH}

5.3.1. Scalability Analysis. To verify the scalability of FLECH, simulations are conducted in the larger AOI of $1000 \mathrm{~m} \times$ $1000 \mathrm{~m}$ area with 1000 nodes. In this case also, BS is located within and outside the AOI. In both cases, FLECH is better than other algorithms in HND condition and FND condition is nearly the same for all the algorithms. Table 9 depicts FND and HND for 1000-node setup. 
TABLE 8: Average energy consumption per round in Scenarios 3 and 4.

\begin{tabular}{lcccccc}
\hline \multirow{2}{*}{ Algorithms } & \multicolumn{2}{c}{200 nodes } & \multicolumn{2}{c}{300 nodes } & \multicolumn{2}{c}{400 nodes } \\
& BS inside AOI & BS outside AOI & BS inside AOI & BS outside AOI & BS inside AOI & BS outside AOI \\
\hline LEACH & 0.2817 & 1.48146 & 0.3965 & 2.06642 & 0.5123 & 2.58728 \\
CHEF & 0.2698 & 1.56976 & 0.3848 & 1.86332 & 0.4915 & 2.14672 \\
ECPF & 0.2870 & 2.05182 & 0.4040 & 2.23108 & 0.5043 & 2.41602 \\
EAUCF & 0.2696 & 1.52638 & 0.3882 & 1.9602 & 0.5011 & 2.25538 \\
MOFCA & 0.2729 & 1.3293 & 0.3911 & 1.7842 & 0.5135 & 2.1075 \\
FLECH & 0.2628 & 1.07176 & 0.3742 & 1.31048 & 0.4846 & 1.5852 \\
\hline
\end{tabular}

TABLE 9

\begin{tabular}{lcccc}
\hline \multirow{2}{*}{ Algorithms } & \multicolumn{2}{c}{ BS within AOI } & \multicolumn{2}{c}{ BS outside AOI } \\
& FND & HND & FND & HND \\
\hline LEACH & 1 & 8 & 1 & 3 \\
CHEF & 1 & 20 & 1 & 4 \\
ECPF & 1 & 7 & 1 & 3 \\
EAUCF & 1 & 14 & 1 & 4 \\
MOFCA & 1 & 14 & 1 & 4 \\
FLECH & 1 & 31 & 1 & 5 \\
\hline
\end{tabular}

5.3.2. Complexity Analysis. There are $n$ numbers of nodes in the network, so it takes maximum of $n$ fuzzy computation for getting the chance value of each node. At the worst case, it may take $(n-1)$ numbers of comparison of chance for a node to get elected as $\mathrm{CH}$. So in total, $n(n-1)$ number of computations occurs for $\mathrm{CH}$ election. In $\mathrm{Big} O$ notation, FLECH complexity is of $O\left(n^{2}-1\right)$ which is again equal to $O\left(n^{2}\right)$.

\section{Conclusion and Future Works}

This paper proposed an algorithm FLECH for clustering nonuniform WSN based on fuzzy logic approach. FLECH improves the WSN network lifetime because it takes into account various important parameters that affect the energy consumption by nodes. It is observed that high residual energy alone is not a suitable parameter for electing $\mathrm{CH}$ in nonuniform node deployment. FLECH combines probabilistic and metric based $\mathrm{CH}$ election techniques with suitable parameters for $\mathrm{CH}$ election in nonuniform WSN. FLECH is simulated along with other clustering algorithms in various scenarios with different network sizes. It is observed from the simulations that when the network size increases FLECH is giving better performance than other algorithms and proves to be scalable. In future, the performance of FLECH may be increased by including more parameters such as coverage redundancy and number of hops between $\mathrm{CH}$ and $\mathrm{BS}$.

\section{Competing Interests}

The authors declare that there is no conflict of interests regarding the publication of this paper.

\section{Acknowledgments}

The authors would like to thank the Department of Science and Technology, India, for their financial support through Fund for Improvement of S\&T Infrastructure (FIST) programme (SR/FST/ETI-349/2013).

\section{References}

[1] I. F. Akyildiz, W. Su, Y. Sankarasubramaniam, and E. Cayirci, "Wireless sensor networks: a survey," Computer Networks, vol. 38, no. 4, pp. 393-422, 2002.

[2] F. Zhao, J. Liu, J. Liu, L. Guibas, and J. Reich, "Collaborative signal and information processing: an information-directed approach," Proceedings of the IEEE, vol. 91, no. 8, pp. 1199-1209, 2003.

[3] A. A. Abbasi and M. Younis, "A survey on clustering algorithms for wireless sensor networks," Computer Communications, vol. 30, no. 14-15, pp. 2826-2841, 2007.

[4] M. M. Afsar and M.-H. Tayarani-N, "Clustering in sensor networks: a literature survey," Journal of Network and Computer Applications, vol. 46, pp. 198-226, 2014.

[5] R. V. Kulkarni, A. Förster, and G. K. Venayagamoorthy, "Computational intelligence in wireless sensor networks: a survey," IEEE Communications Surveys and Tutorials, vol. 13, no. 1, pp. 68-96, 2011.

[6] L. A. Zadeh, "Fuzzy sets," Information and Computation, vol. 8, no. 3, pp. 338-353, 1965.

[7] L. A. Zadeh, "Outline of a new approach to the analysis of complex systems and decision processes," IEEE Transactions on Systems, Man and Cybernetics, no. 1, pp. 28-44, 1973.

[8] W. B. Heinzelman, A. P. Chandrakasan, and H. Balakrishnan, "An application-specific protocol architecture for wireless microsensor networks," IEEE Transactions on Wireless Communications, vol. 1, no. 4, pp. 660-670, 2002.

[9] O. Younis and S. Fahmy, "HEED: a hybrid, energy-efficient, distributed clustering approach for ad hoc sensor networks," IEEE Transactions on Mobile Computing, vol. 3, no. 4, pp. 366379, 2004.

[10] I. Gupta, D. Riordan, and S. Sampalli, "Cluster-head election using fuzzy logic for wireless sensor networks," in Proceedings of the 3rd Annual Communication Networks and Services Research Conference, pp. 255-260, IEEE, Nova Scotia, Canada, May 2005.

[11] J.-M. Kim, S.-H. Park, Y.-J. Han, and T.-M. Chung, "CHEF: Cluster Head Election mechanism using Fuzzy logic in wireless sensor networks," in Proceedings of the 10th International Conference on Advanced Communication Technology, pp. 654-659, Gangwon-Do, South Korea, February 2008. 
[12] H. Taheri, P. Neamatollahi, O. M. Younis, S. Naghibzadeh, and M. H. Yaghmaee, "An energy-aware distributed clustering protocol in wireless sensor networks using fuzzy logic," Ad Hoc Networks, vol. 10, no. 7, pp. 1469-1481, 2012.

[13] H. Bagci and A. Yazici, "An energy aware fuzzy approach to unequal clustering in wireless sensor networks," Applied Soft Computing Journal, vol. 13, no. 4, pp. 1741-1749, 2013.

[14] H. Bagci and A. Yazici, "An energy aware fuzzy unequal clustering algorithm for wireless sensor networks," in Proceedings of the 6th IEEE World Congress on Computational Intelligence (WCCI '10), IEEE, July 2010.

[15] S. A. Sert, H. Bagci, and A. Yazici, "MOFCA: multi-objective fuzzy clustering algorithm for wireless sensor networks," Applied Soft Computing Journal, vol. 30, pp. 151-165, 2015.

[16] E. H. Mamdani, "Application of fuzzy logic to approximate reasoning using linguistic synthesis," IEEE Transactions on Computers, vol. 26, no. 12, pp. 1182-1191, 1977. 


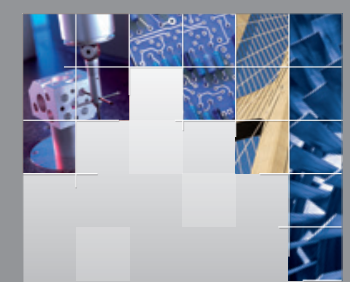

\section{Enfincering}
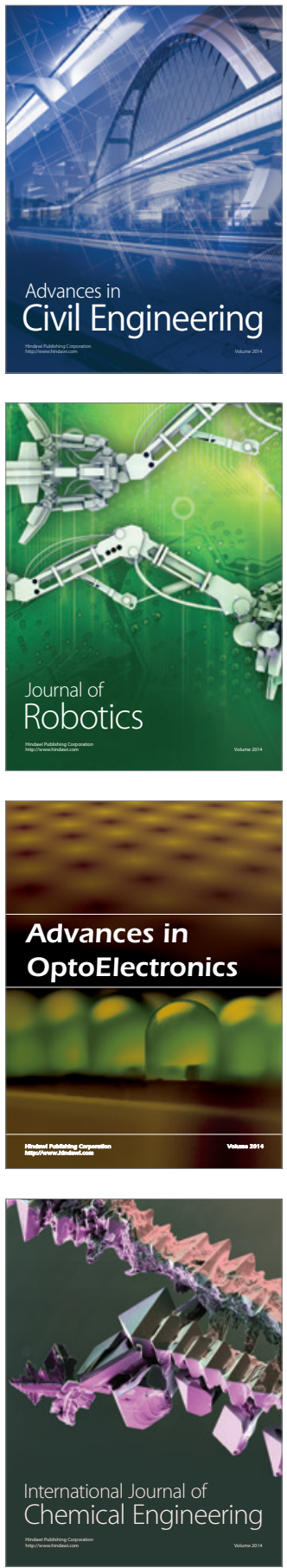

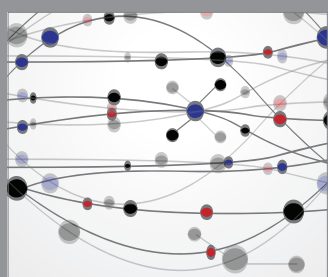

The Scientific World Journal

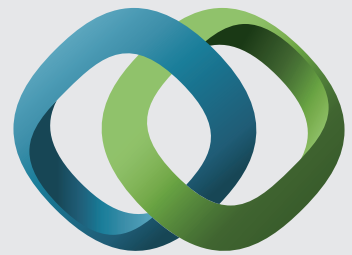

\section{Hindawi}

Submit your manuscripts at

https://www.hindawi.com
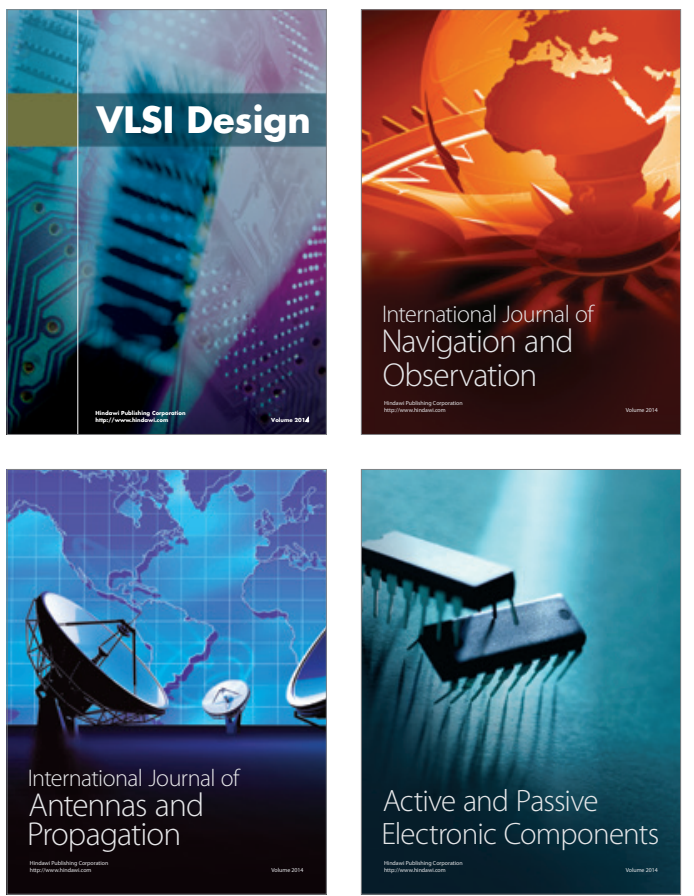
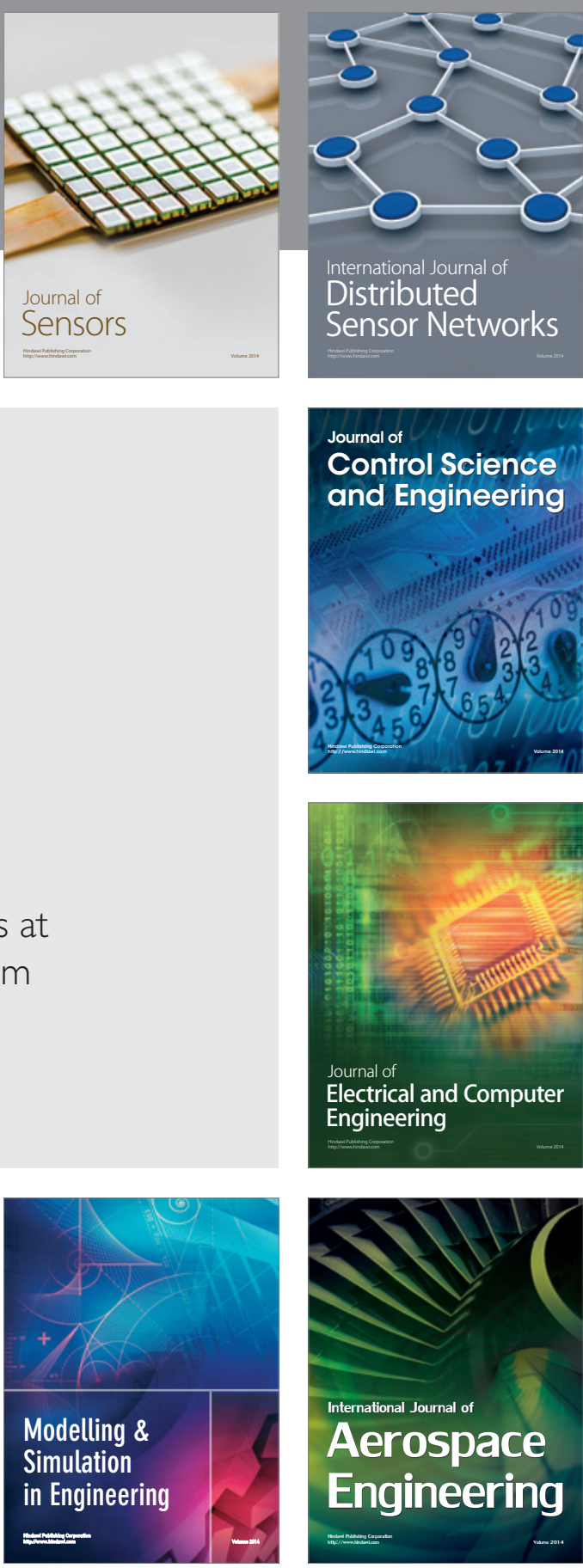

International Journal of

Distributed

Sensor Networks

$-$

Joumal of

Control Science

and Engineering
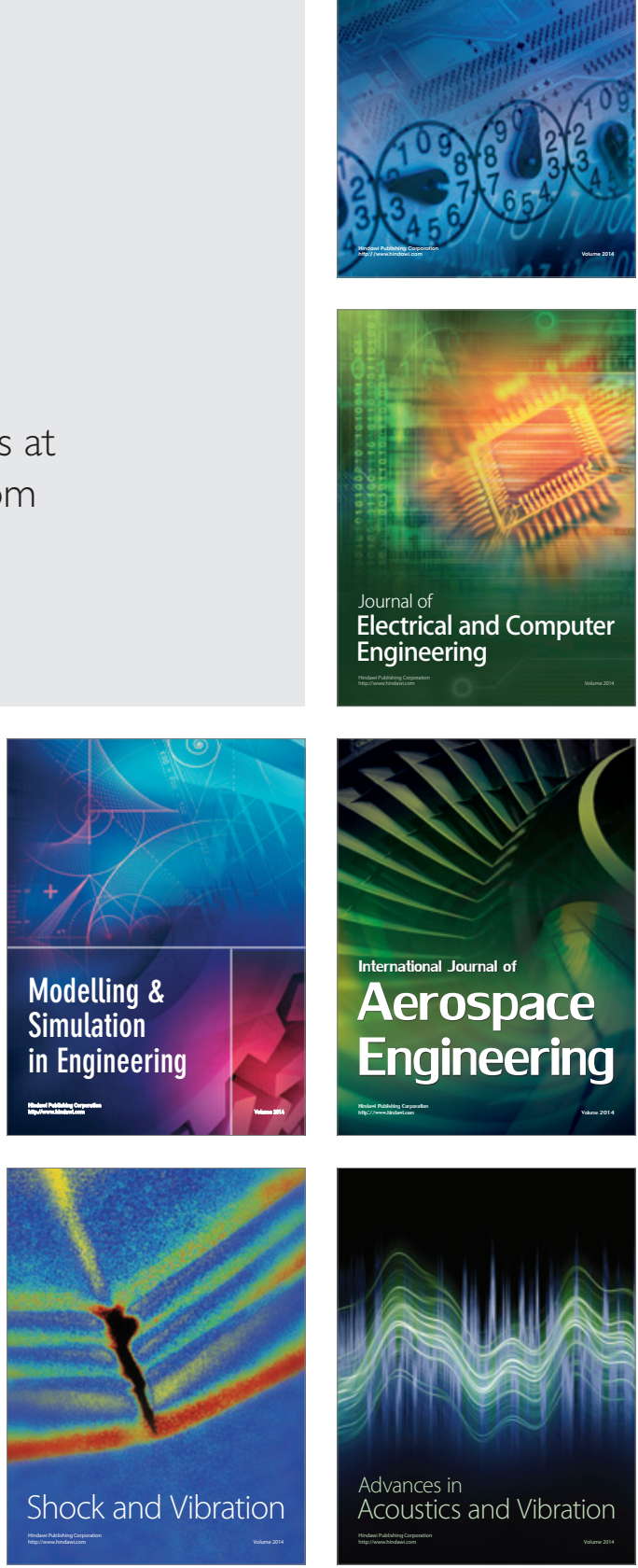